\title{
Breast Cancer Mimic: Cutaneous B-Cell Lymphoma Presenting as an Isolated Breast Mass
}

\author{
Margaret Taghavi $^{\mathrm{a}}$ Yanhong Zhang $^{\mathrm{b}}$ Karen Lindfors $^{\mathrm{c}}$ \\ Shadi Aminololama-Shakeri ${ }^{\mathrm{c}}$ \\ ${ }^{a}$ Department of Radiology, UC San Francisco Medical Center, San Francisco, Calif., and \\ Departments of ${ }^{\mathrm{b}}$ Pathology and ${ }^{\mathrm{C}}$ Radiology, UC Davis Medical Center, \\ Sacramento, Calif., USA
}

\section{Key Words}

Primary cutaneous B-cell lymphoma · Breast mass - Lymphoma

\begin{abstract}
Background: Primary cutaneous B-cell lymphoma typically localizes to the skin, and dissemination to internal organs is rare. Lymphomatous involvement of the breasts is also rare. We describe the clinical and radiological findings of an unusual case of primary cutaneous B-cell lymphoma presenting as an isolated breast mass without associated skin changes. Case Presentation: The patient was a 55-year-old Caucasian female who initially presented with cutaneous B-cell lymphoma around her eyes and forehead with recurrence involving the skin between her breasts. Three years after terminating treatment due to a lack of symptoms, she presented for an annual screening mammogram that found a new mass in her upper inner right breast without imaging signs of cutaneous extension. On physical examination, there were no corresponding skin findings. Due to the suspicious imaging features of the mass that caused concern for primary breast malignancy, she underwent a core biopsy which revealed cutaneous B-cell lymphoma. Conclusion: When evaluating patients with a systemic disease who present with findings atypical for that process, it is important to still consider the systemic disease as a potential etiology, particularly with lymphoma given its reputation as a great mimicker.

(c) 2014 S. Karger AG, Basel
\end{abstract}


Taghavi et al.: Breast Cancer Mimic: Cutaneous B-Cell Lymphoma Presenting as an Isolated Breast Mass

\section{Background}

Primary cutaneous B-cell lymphoma (pCBCL) typically localizes to the skin, and dissemination to internal organs is rare. Hodgkin's and non-Hodgkin's lymphomatous involvement of the breasts is also rare. We discuss the clinical and radiological findings of a case of CBCL presenting as an isolated breast mass without associated skin changes, which has not been reported to date.

\section{Case Report}

In 1993, a 36-year-old Caucasian female with a history of dyslipidemia, gastroesophageal reflux, and fibromyalgia presented to her dermatologist with redness around her eyes and forehead. Biopsy of this area demonstrated cutaneous marginal zone B-cell lymphoma (CMZL). She was treated with local radiation therapy and topical nitrogen mustard with control of symptoms for 2 years until it recurred locally. Low-dose radiation to the same area was repeated. She was also treated with Rituxan, Cytoxan, Vincristine and Prednisone for 3 months but had a recurrence with new cutaneous lesions involving the skin between her breasts and on her back. She participated in a series of experimental clinical trials with partial response. Since 2009, she had not received any further treatment given the low-grade nature of the lymphoma and lack of symptoms.

\section{Imaging Findings}

In 2012, the patient underwent a routine annual screening mammogram, which found a new 18-mm mass in the upper inner right breast at approximately 2 o'clock on a background of scattered fibroglandular tissue (fig. 1a). Given the patient's known history of CBCL, the primary care physician was asked to evaluate for skin findings in this area, of which there were none.

The right breast mass was further evaluated with diagnostic mammographic views with spot compression. These confirmed a partially circumscribed, partially obscured mass at 2 o'clock in the posterior third of the right breast. There were no suspicious microcalcifications, architectural distortion, or mammographic signs of skin changes. On physical examination, the breast imager confirmed that there were no associated cutaneous findings.

Targeted high-resolution ultrasound of the right breast at 2 o'clock showed a $13 \times 15 \times$ $6 \mathrm{~mm}$ ill-defined oval hypoechoic heterogeneous mass oriented parallel to the skin surface, with the center of the mass approximately 6-7 mm below the skin surface. There was no cutaneous extension seen on ultrasound (fig. 1b). The ill-defined and angulated margins of the mass caused concern for a primary breast carcinoma. Because this mass was new and demonstrated suspicious imaging features, the patient underwent ultrasound-guided biopsy. Pathology was consistent with CMZL (fig. 2).

A 2-[fluorine-18]fluoro-2-deoxy-D-glucose (18F-FDG) positron emission tomography (PET)/computed tomography (CT) scan was performed to evaluate for other lesions. A $16 \times$ $12 \mathrm{~mm}$ mass in the upper inner right breast, anterior depth, was demonstrated to be metabolically active with a maximum standardized uptake value (SUVmax) of $3.1 \mathrm{~g} / \mathrm{ml}$ (fig. 3). This represented the mammographic and sonographic correlate. The PET/CT also showed metabolically active left axillary, right inguinal and right iliac lymph nodes. There were no metabolically active right axillary lymph nodes or cutaneous lesions. 
Taghavi et al.: Breast Cancer Mimic: Cutaneous B-Cell Lymphoma Presenting as an Isolated Breast Mass

Due to the overall minimal disease on PET/CT, lack of B symptoms, and the low-grade nature of the lymphoma, the patient did not meet the criteria for additional treatment and is being followed up clinically.

\section{Discussion}

Breast Lymphoma

Hodgkin's and non-Hodgkin's lymphomatous involvement of the breast is extremely rare but represents the largest group of tumors that metastasize to the breast [1-5]. Approximately $0.15 \%$ of malignant mammary neoplasms are lymphomas and less than $0.5 \%$ of all malignant lymphomas primarily involve the breast $[1-3,6]$. The small amount of lymphoid tissue in the breast accounts for the rarity of primary breast lymphoma.

Breast lymphomas most frequently manifest as an upper outer quadrant breast mass, in the typical location of intramammary lymph nodes. There may be skin changes, such as skin fixation, and cutaneous inflammatory changes, like skin thickening [1-3]. While breast cancers occur more frequently in the left breast $[1,2]$, unilateral primary and secondary breast lymphoma is predominantly right-sided $[1,2,5,6]$. Mammary lymphomas also tend to be larger at diagnosis than primary breast malignancies [1].

The most common mammographic feature of breast lymphoma is a round or oval mass with indistinct or circumscribed margins. Less frequently, breast lymphomas present as global asymmetry on mammography. Breast lymphomas typically do not demonstrate microcalcifications or spiculated margins that are associated with primary breast carcinoma.

Sonographic features of breast lymphoma are nonspecific. They can present as a heterogeneously hypoechoic mass with indistinct margins, circumscribed hypoechoic mass, or diffuse nodular infiltration of the breast, which is more common in women with extensive involvement of both breasts. Breast lymphomas on ultrasound can demonstrate hypervascularity but do not demonstrate posterior acoustic shadowing associated with breast carcinoma $[2,3,7,8]$.

Magnetic resonance imaging of breast lymphoma demonstrates a hypointense or isointense mass on T1-weighted sequences and hyperintense mass on T2-weighted sequences. On post-contrast images, there is heterogeneous enhancement with rapid initial increase and washout kinetics on dynamic contrast-enhanced images [2].

PET/CT is the imaging modality of choice for staging and follow-up of patients with extranodal involvement for Hodgkin's lymphoma and most cases of non-Hodgkin's lymphoma. Imaging of tumor metabolism with 18F-FDG PET identifies sites of disease that may not be appreciable on CT. The intensity of FDG uptake characterizes tumor activity. Breast lymphomatous involvement is seen as increased FDG uptake [9].

\section{Primary Cutaneous B-Cell Lymphoma}

pCBCLs are B-cell lymphomas that originate in the skin and present without extracutaneous disease. pCBCLs constitute approximately 25-29\% of all primary cutaneous lymphomas in the United States, more commonly affecting men than women, with incidence increasing with age. pCBCL is almost exclusively a disease of non-Hispanic whites [10].

There are three types of pCBCL: primary CMZL (pCMZL), primary cutaneous follicle center lymphoma, and primary cutaneous diffuse large B-cell lymphoma, leg type. The two former subtypes are recognized as indolent lymphomas, while the latter has a more intermediate behavior $[10,11]$. The following discussion will focus on PCMZL, which is the subtype of pCBCL in our patient. pCMZL has only recently been recognized as a distinct 
clinical entity [12]. The tumor cells originate in the 'marginal zone' of the secondary lymphoid follicles in the skin [13].

Patients with pCMZL typically present with solitary or clustered reddish or red brown papules, nodules and plaques sometimes surrounded by an erythematous halo [14]. It has a predilection for the trunk and upper extremities. While it has frequent cutaneous relapses, it rarely causes extracutaneous dissemination [8, 10,12]. pCMZL is associated with an excellent prognosis with 5-year survival rates ranging from 89 to $99 \%[4,10,12,15]$.

pCMZL responds well to local radiotherapy or excision. Topical therapies such as corticosteroids, topical nitrogen mustard, cryotherapy, intralesional tramcinolone, and intralesional interferon are frequently used with success in pCMZL. Chlorambucil and intravenous or intralesional rituximab have also been used in patients with multifocal lesions untreatable by radiation therapy [10].

\section{Conclusions}

We present an unusual case of CBCL manifesting as an isolated breast mass without associated skin changes. We are the first to describe its imaging features given that CBCL is typically followed clinically by physical exam. While Hodgkin's lymphoma and nonHodgkin's lymphoma are known to be great mimickers of other diseases, CBCL is less known to present similarly to other maladies. Our case report is a reminder that when evaluating patients with a systemic disease who present with findings atypical for that process, the systemic disease should still be considered as a potential etiology, particularly with lymphoma.

\section{Disclosure Statement}

The authors declare that they have no competing interests.

\section{References}

1 Brustein S, Filippa DA, Kimmel M, et al: Malignant lymphoma of the breast: a study of 53 patients. Ann Surg 1987;205:144-150.

2 Yang WT, Lane DL, Le-Petross HT, et al: Breast lymphoma: imaging findings of 32 tumors in 27 patients. Radiol 2007;245:692-702.

-3 Liberman L, Giess CS, Dershaw DD, et al: Non-Hodgkin lymphoma of the breast: imaging characteristics and correlation with histopathologic findings. Radiology 1994;192:157-160.

4 Bailey EM, Ferry JA, Harris NL, et al: Marginal zone lymphoma (low-grade B-cell lymphoma of mucosaassociated lymphoid tissue type) of skin and subcutaneous tissue: a study of 15 patients. Am J Surg Pathol 1996;20:1011-1023.

5 Loughrey MB, Windrum P, Catherwood MA, et al: WHO reclassification of breast lymphomas. J Clin Pathol 2004;57:1213-1214.

6 Topalovski M, Crisan D, Mattson JC: Lymphoma of the breast. A clinicopathologic study of primary and secondary cases. Arch Pathol Lab Med 1999;123:1208-1218.

7 Yang WT, Muttarak M, Ho LW: Nonmammary malignancies of the breast: ultrasound, CT and MRI. Semin Ultrasound CT MR 2000;21:375-394.

-8 Surov A, Holzhausen HJ, Wienke A, et al: Primary and secondary breast lymphoma: prevalence, clinical signs and radiologic features. Br J Radiol 2012;85:e195-e205.

9 Paes FM, Kalkanis DG, Sideras PA, et al: FDG PET/CT of extranodal involvement in non-Hogkin lymphoma and Hodgkin disease. Radiographics 2010;30:269-291.

10 Suàrez AL, Querfeld C, Horwitz S, et al: Primary cutaneous B-cell lymphomas: part II. Therapy and future directions. J Am Acad Dermatol 2013;69:343.e1-11. 


\section{Case Reports in Oncology}

\begin{tabular}{l|l}
\hline Case Rep Oncol 2014;7:685-691 & \\
\hline DOI: $10.1159 / 000368043$ & $\begin{array}{l}\text { ○ 2014 S. Karger AG, Basel } \\
\text { www.karger.com/cro }\end{array}$ \\
\hline
\end{tabular}

Taghavi et al.: Breast Cancer Mimic: Cutaneous B-Cell Lymphoma Presenting as an Isolated Breast Mass

11 Wilcox, RA: Cutaneous B-cell lymphomas: 2013 update on diagnosis, risk-stratification, and management. Am J Hematol 2013;88:73-76.

12 Pandolfino TL, Siegel RS, Kuzel TM, et al: Primary cutaneous B-cell lymphoma: review and current concepts. J Clin Oncol 2000;18:2152-2168.

13 Thieblemont C: Clinical presentation and management of marginal zone lymphomas. Hematology Am Soc Hematol Educ Program 2005:307-313.

14 Cerroni L, Signoretti S, Höfler G, et al: Primary cutaneous marginal zone B-cell lymphoma: a recently described entity of low-grade malignant cutaneous B-cell lymphoma. Am J Surg Pathol 1997;21:1307-1315.

15 Hoefnagel JJ, Vermeer MH, Jansen PM, et al: Primary cutaneous marginal zone B-cell lymphoma: clinical and therapeutic features in 50 cases. Arch Dermatol 2005;141:1139-1145. 


\section{Case Reports in Oncology}

\begin{tabular}{l|l}
\hline Case Rep Oncol 2014;7:685-691 \\
\hline DOI: 10.1159/000368043 & $\begin{array}{l}\text { (c) 2014 S. Karger AG, Basel } \\
\text { www.karger.com/cro }\end{array}$ \\
\hline
\end{tabular}

Taghavi et al.: Breast Cancer Mimic: Cutaneous B-Cell Lymphoma Presenting as an Isolated Breast Mass

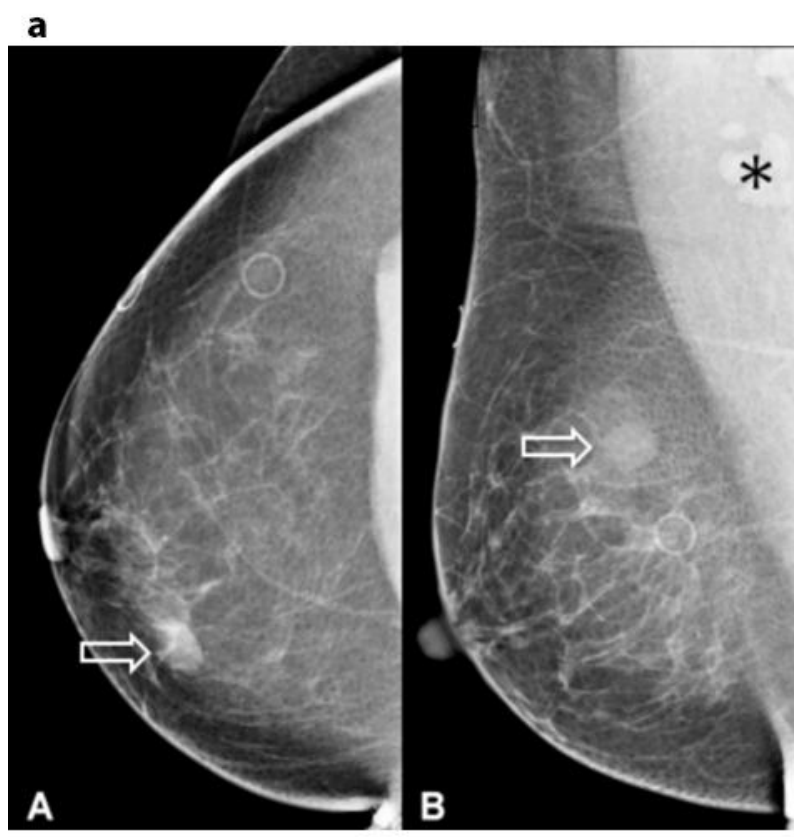

b

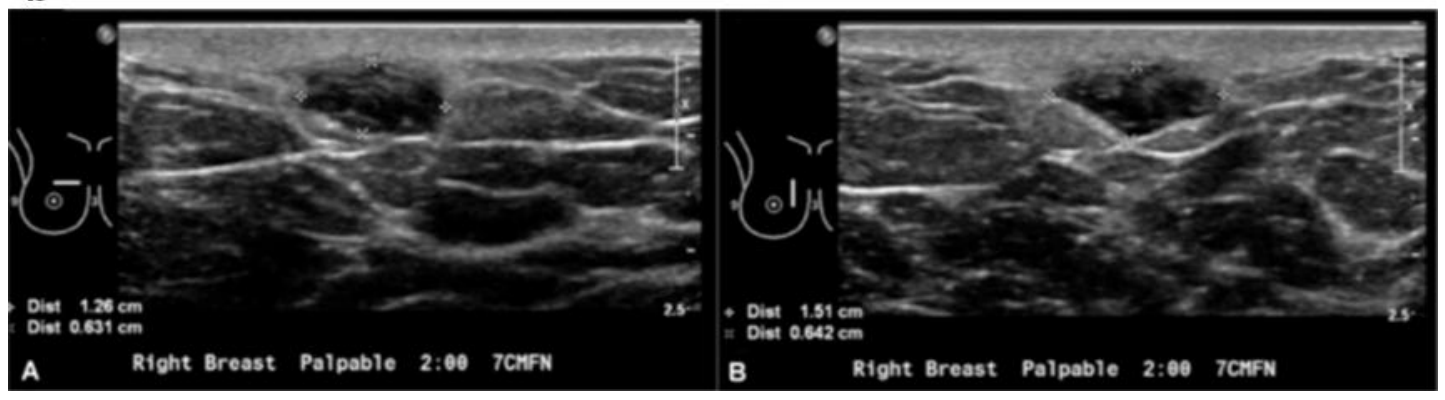

Fig. 1. a A 55-year-old female presented for a routine craniocaudal (A) and mediolateral oblique (B) screening mammogram, which found a new 18-mm mass (arrows) in the posterior third of the right breast at approximately 2 o'clock on a background of scattered fibroglandular tissue. There were no suspicious microcalcifications, architectural distortion or mammographic signs of skin changes. Note that there is no mole marker over the mass to indicate skin findings. Small, non-enlarged right axillary lymph nodes were present (asterisk). b The 55-year-old female presented for a high resolution targeted ultrasound of the right breast. A physical exam prior to the breast ultrasound found no skin changes in the upper inner right breast. Targeted ultrasound of the right breast in the transverse (A) and longitudinal (B) planes showed a heterogeneously hypoechoic $13 \times 15 \times 6 \mathrm{~mm}$ mass with ill-defined, angulated margins located approximately 6-7 mm below the skin surface. The mass did not extend to the skin surface on ultrasound. Because the margins of the mass caused concern for a breast malignancy, an ultrasound-guided biopsy was recommended. 


\section{Case Reports in Oncology}

\begin{tabular}{l|l}
\hline Case Rep Oncol 2014;7:685-691 \\
\hline DOI: 10.1159/000368043 & $\begin{array}{l}\text { (C) 2014 S. Karger AG, Basel } \\
\text { www.karger.com/cro }\end{array}$ \\
\hline
\end{tabular}

Taghavi et al.: Breast Cancer Mimic: Cutaneous B-Cell Lymphoma Presenting as an Isolated Breast Mass

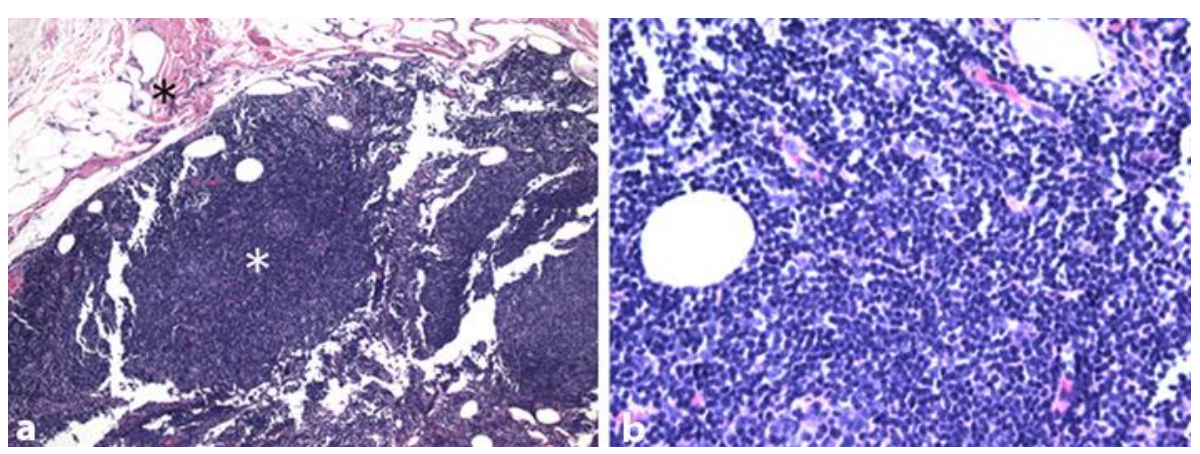

Fig. 2. Histopathologic features of CMZL. a Hematoxylin-eosin staining shows nodular to diffuse lymphocytic infiltrate (white asterisk) involving fibroadipose tissue (black asterisk). b The diffuse lymphocytic infiltrate is composed primarily of small mature-appearing lymphocytes.

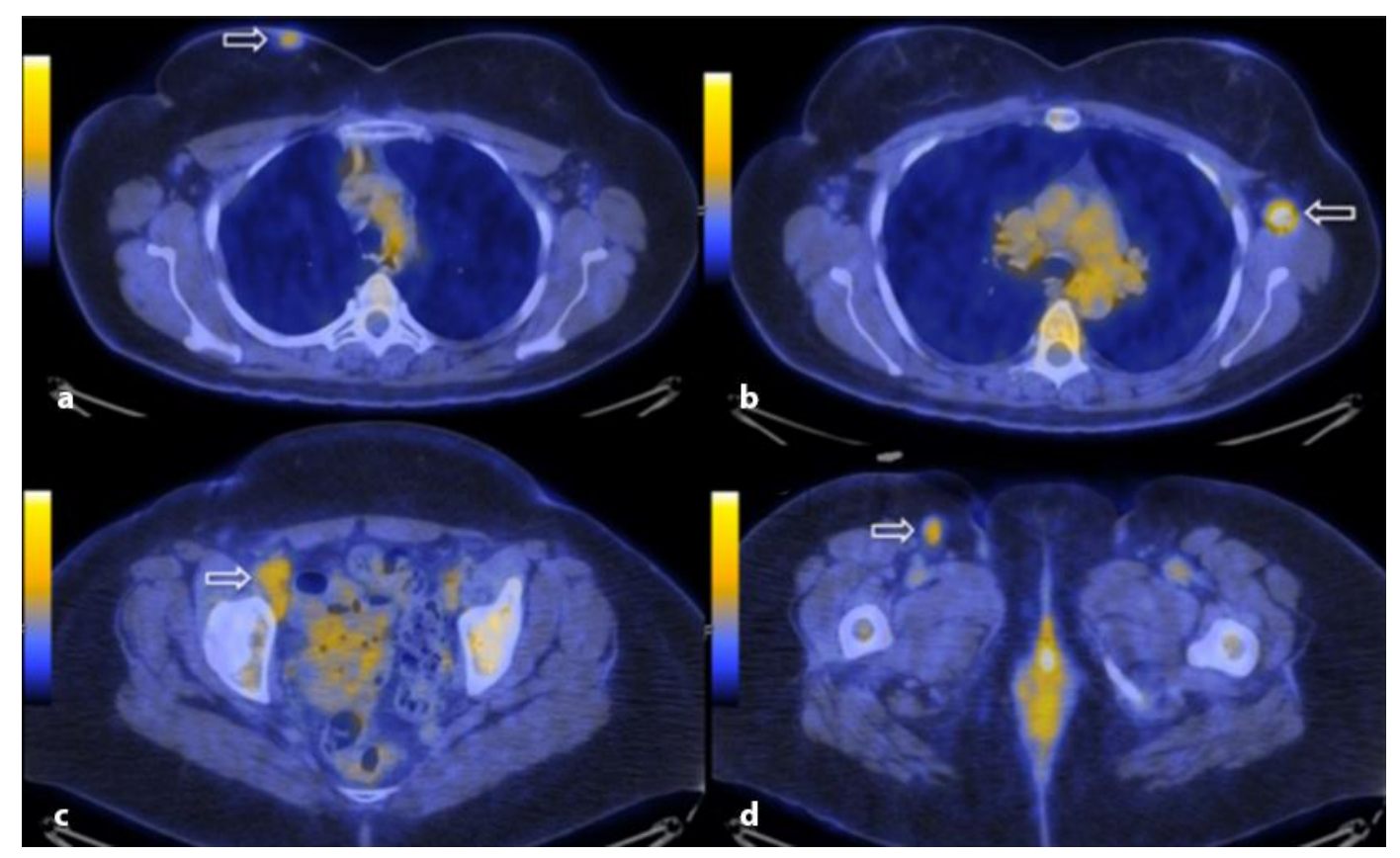

Fig. 3. The 55-year-old female with CBCL, originally involving the face, presented for an FDG-PET/CT. a Fused PET/CT images showed a $16 \times 12 \mathrm{~mm}$ mass in the upper inner right breast, anterior depth, with an SUVmax of $3.1 \mathrm{~g} / \mathrm{ml}$. There was no overlying skin thickening or hypermetabolic activity. b A hypermetabolic $16 \times 24 \mathrm{~mm}$ left axillary lymph node was also present with an SUVmax of $8.1 \mathrm{~g} / \mathrm{ml}$. There were no hypermetabolic right axillary lymph nodes. c A hypermetabolic right external iliac lymph node was present, measuring $15 \times 24 \mathrm{~mm}$ with an SUVmax of $3.9 \mathrm{~g} / \mathrm{ml}$. d Several right inguinal lymph nodes were also seen, the largest measuring $12 \times 17 \mathrm{~mm}$ with an SUVmax of $3.7 \mathrm{~g} / \mathrm{ml}$. 